\title{
Remarks on the possibility of introducing the fractionated dose of the inactivated poliomyelitis vaccine in the Latin American Child Immunization Schedule*
}

\author{
Antonio Arbo', Luiza Helena Falleiros-Arlant², Eduardo L. López³, José Brea del Castillo4, \\ Celia Martínez de Cuellar', Gabriela Moreno5, Roger Rolón', Javier Cerda', Salim Eguiazú \\ and Study Group of Sociedad Paraguaya de Pediatría
}

\begin{abstract}
Given that the last notified case of poliomyelitis due to wild poliovirus type 2 was in 1999, in 2012, the Strategic Advisory Group of Experts on Immunization (SAGE) of the World Health Organization (WHO) recommended the withdrawal of the type 2 component of oral polio vaccine (OPV) and the introduction of a bivalent OPV (bOPV) in all countries by 2016. WHO recommended also that the withdrawal should be preceded by the introduction of at least one dose of inactivated poliovirus vaccine (IPV) in routine immunization schedules. The introduction of IPV prior to the change of the bOPV in 2016 to trivalent OPV (tOPV) was based on the concept of ensuring that a substantial proportion of the population would be protected against type 2 polio after the removal of the type 2 OPV. However, the world's two producers of IPV (Bilthoven Biologicals and Sanofi) have faced problems in the production of this vaccine and therefore reported a reduction of the global supply of IPV. In response to the potential shortage of IPV, at a meeting held on March 10 2017, the SAGE and Technical Advisory Group (TAG) of the Pan American Health Organization (PAHO) urged the countries in the Latin American region to replace the routine administration of the full doses of inactivated polio vaccine (IPV-C) in the immunization schedule (administered by intramuscular route), administering a fraction of the full dose in two intradermal shots (IPV-f). The possibility of this strategy was analyzed by opinion leaders convened by the Paraguayan Society of Pediatrics with the support of the Latin American Society of Pediatric Infectious Diseases (SLIPE) and Latin American Association of Pediatrics (ALAPE). This document presents the results of the discussion.
\end{abstract}

\section{Introduction}

$\mathrm{T}$ his document is the result of a meeting held in Asunción, Paraguay, on November 17 and 18 2017. The meeting was an initiative of the Paraguayan Society of Pediatrics, with the support of the Latin American Society of Pediatric Infectious Disease (SLIPE) and the Latin American Association of Pediatrics (ALAPE) in response to the recommendation of PAHO's Technical Advisory Group (TAG), dated March $102017^{1}$, which urged the countries of the region to replace the usual administration of the complete dose of the inactivated poliovirus (IPV-C) vaccine (administered intramuscularly) with the administration of a fraction of the complete dose (1/5 of the dose) intradermally (IPV-f) in their immunization schedules. This recommendation came up as an answer to the possibility of running short in production. World producers are facing problems and have therefore announced several reductions in the global supply of the inactivated polio vaccine (IPV), antici- pating a global shortage of this vaccine that will persist and worsen at least until late $2018^{1,2}$.

\section{Current situation of poliomyelitis worldwide and in the Americas}

In the late 80 's, the World Health Assembly decided to eradicate poliomyelitis, the leading cause of irreversible paralysis (predominantly involving the lower limbs) in children ${ }^{3}$. Since then, the number of cases of symptomatic infection caused by the wild poliovirus (WPV) has dropped by $>99.9 \%$, going from about 350,000 cases of polio estimated in 1988 to 37 cases reported in three countries in 2016 and 22 cases in two countries (Afghanistan and Pakistan) in 2017 and 8 cases as of May 29, 2018, also in Afghanistan and Pakistan ${ }^{4}$. In 1985, all the countries in the American continent set the target of eradicating polio from the Region, and the last case of polio in the Americas was reported in Junin, Peru, on August 23, 19915. In 1994, the Americas Region was certified as being free from the circulation of the wild poliovi-

\author{
'Paraguay. \\ ${ }^{2}$ Brazil. \\ ${ }^{3}$ Argentina. \\ ${ }^{4}$ República Dominicana. \\ ${ }^{5}$ Chile. \\ *Document prepared in June \\ 2018. A Spanish version was \\ published by Revista Chilena de \\ Infectología as a Supplement \\ in October, 2018 and can be \\ consulted in: http://www.revinf. \\ cl/index.php/revinf/issue/archive \\ Corresponding authors: \\ Antonio Arbo Sosa \\ antonioarbo@hotmail.com \\ Luiza Helena Falleiros-Arlant \\ luizahfc@terra.com.br
}


rus $^{6}$. This reduction was primarily achieved through the use of a trivalent oral poliovirus (tOPV) vaccine, which contains live attenuated polioviruses of types 1,2 and $3^{2}$.

\section{$O P V$ vaccine and risk of flaccid paralysis}

The use of the tOPV vaccine is not free of risks. The vaccine can be associated with disease, causing cases of poliomyelitis due to the vaccine-associated virus (VAPP), but in addition, any component can revert its attenuation and cause paralysis; there may be cases of polio resulting from a vaccine-derived poliovirus (VDPV), when the attenuated quality reverts through genetic mutation. The risk of VAPP varies from country to country in Latin America, ranging from 1 case per 2.56 to 4.1 million doses applied ${ }^{7}$. The disease caused by the mutated virus from the vaccine has the same clinical presentation, severity and case fatality ratio as polio caused by the wild virus. Our continent experienced a VDPV outbreak in 2001, on the Island of Hispaniola, with 21 confirmed cases due to a mutation of serotype 1 of the attenuated vaccine ${ }^{8}$. In the last few decades, more than 500 cases of VDVP have been reported in the world. Almost all (97\%) the outbreaks of VDPV that occurred in

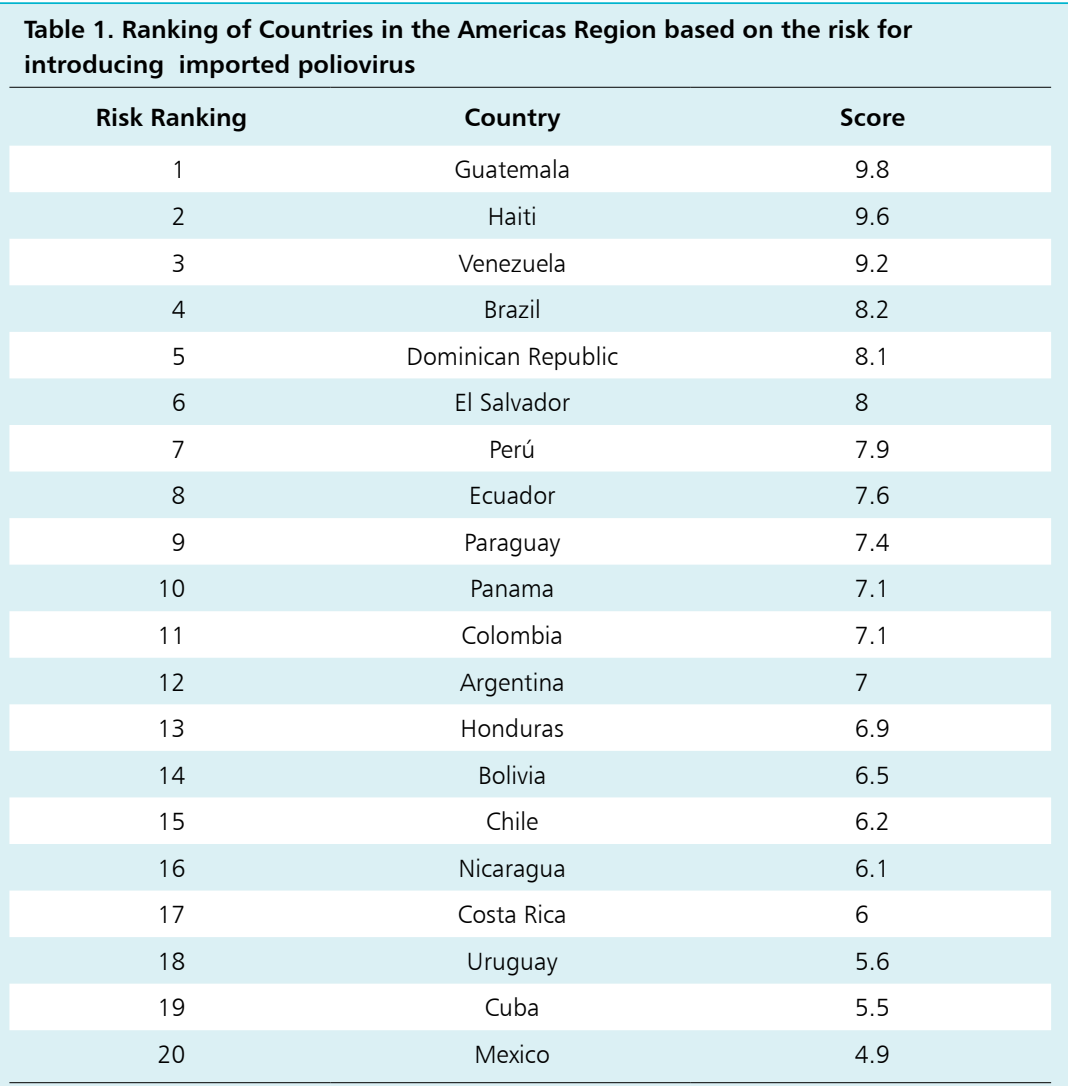

Source: PAHO. Technical Advisory Group on Vaccine-preventable Diseases (TAG). XXIV Meeting 12-14 July 2017 recent years have been caused by a virus derived from OPV type 2 (4). It is estimated that for every case that is reported case, there are from 100 to 1,000 people eliminating the virus into the environment despite being asymptomatic ${ }^{9}$.

Even when since 2000 the United States of America (USA) and later on most European countries have only used the IPV ${ }^{10}$ that contains the three types of poliovirus $1,2,3$, the vast majority of countries in Latin America, Africa and Asia continued to use the tOPV vaccine to protect against the disease until April 2016. Yet, in 2014, the Latin American Society of Pediatric Infectology (SLIPE) and the Latin American Association of Pediatrics (ALAPE) had recommended using the IPV to replace OPV, IPV given that the risk of vaccine-related disease exceeds the risk of disease by the natural acquisition of wild poliovirus ${ }^{11}$.

\section{Situation of the Latin America and Caribbean (LAC) Region regarding the risk of introducing an imported poliovirus}

Transmission of the wild polio virus continues to occur in Afghanistan and Pakistan in 20184. If transmission is not stopped in these last remaining redoubts, there could be up to 200,000 new cases annually in 10 years worldwide ${ }^{12}$. Although migration rates from these countries are very low in Latin America, the rate of immigration from African countries (mainly from Angola to Brazil) is not negligible, which means that this region is not exempt from the risk of introducing imported poliovirus ${ }^{13}$. PAHO conducted a risk analysis of the LAC countries using four components, including: 1) immunization coverage rates, 2) surveillance of Acute Flaccid Paralysis, 3) history of outbreaks or evidence of circulation of VDPV and preparedness for response, and 4) national capacity to detect the introduction of wild polio virus or VDPV-related events, and developed a risk scale that ranges from 0 (minimum risk) to 10 (high risk) based on those four elements ${ }^{14}$. Several countries in the Region present a moderate or very high risk; such is the case of Venezuela in northern South America, whereas in the Southern Cone there are concerning levels of risk in Brazil and Paraguay (Table 1). Therefore, a thorough analysis of these aspects is warranted when determining the polio immunization strategy.

\section{Reasons for the removal of the polio 2 component of the tOPV vaccine and for the introduction of IPV in the primary vaccination scheme}

Wild type 2 poliovirus has not circulated naturally since 1999 (the last case was reported in Aligarh, India), which obviates the need for the type 2 component in the vaccine ${ }^{2}$. Moreover, the presence of the type 2 component in the vaccine hampers the immune response 
to polio viruses type 1 and 3 ; therefore, the number of doses required to reach the herd immunity thresholds for these two serotypes is higher with tOPV than with the bivalent vaccine that contains only serotypes 1 and 3 (bOPV) ${ }^{15}$.

The $65^{\text {th }}$ World Health Assembly (May 2012) approved the recommendation "to complete the eradication of the polio virus as a global public health programmatic emergency"16. In the Formulation of the Strategic Plan for the Eradication of Poliomyelitis and the 20132018 final phase for a polio-free world, the WHA urged the discontinuation of the tOPV globally; they recommended starting the change with OPV type 2 and encouraged to switch from tOPV to bOPV ${ }^{2}$. However, due to the persistence of the risk of paralysis associated with the circulating type 2 poliovirus (a risk that is estimated to persist until 5 years after stopping the administration of tOPV), it was deemed necessary to keep the population immunized against poliovirus serotype 2 with an immunogen that contains the inactivated form of the virus, which is not shed into the environment and poses no risks of mutation in the intestine. Therefore, countries that implement the bOPV as a risk-mitigation measure must introduce at least one dose of the IPV ${ }^{16}$. Introducing IPV before switching from tOPV to bOPV ensures the protection of a substantial proportion of the population against poliovirus serotype 2 after the withdrawal of OPV type 2, and thus mitigates the risks of reintroduction of type 2 , while enhancing immunity against serotypes 1 and 3 .

\section{Proposal for replacing IPV-C with IPV-f}

When The Polio Eradication \& Endgame Strategic Plan 2013-2018 was released in 2013, it was already anticipated that the production of IPV might fail to meet the increased demand ${ }^{2}$. In 2014, the production of IPV had fallen by $40 \%$ and the problems were expected to persist for more than 5 years, a potential improvement was not foreseen until 2020. To address that issue, the group of experts of WHO and PAHO suggested that "countries (should) consider the adoption of fractional doses of intradermal inactivated polio vaccine"2,14,15. This recommendation -although not specified in the document- would apply for countries in Latin America, Africa and Asia and not for the US and Europe. The immunization schedule suggested is to administer IPV-f in the first two doses ( 2 and 4 months) and to use bOPV from then on (Box 1).

\begin{tabular}{|l|c|c|c|c|c|}
\hline Box 1. Polio vaccination schedule as proposed by PAHO's TAG \\
\hline Vaccination & \multicolumn{3}{|c|}{ Primary } & \multicolumn{2}{c|}{ Booster } \\
\cline { 2 - 6 } Schedule & $\begin{array}{c}\text { 1st. } \\
\text { dose }\end{array}$ & $\begin{array}{c}\text { 2nd. } \\
\text { dose }\end{array}$ & $\begin{array}{l}\text { 3rd. } \\
\text { dose }\end{array}$ & $\begin{array}{c}\text { 1st. } \\
\text { booster }\end{array}$ & $\begin{array}{c}\text { 2nd. } \\
\text { booster }\end{array}$ \\
\cline { 2 - 6 } & IPV-f & IPV-f & bOPV & bOPV & bOPV \\
\hline
\end{tabular}

\section{Immunogenicity of the IPV-f vaccine}

As part of the response to the IPV shortage, PAHO/ WHO recommended to implement the intradermal administration of one fraction of the complete IPV vaccine, specifically one fifth of the complete dose administered intramuscularly. Multiple studies have evaluated the immunogenicity of IPV administered in fractional doses (IPV-f) via that route and compared it against the IM application of the usual or complete doses of IPV (IPV-C). In a randomized study conducted in Cuba, Resik S. et al ${ }^{17}$ compared the immunogenicity of IPV-f (one fifth of a complete dose) administered intradermally with a special device (Bioejector 2000, Bioject) with that of IM IPV-C. They assessed 310 four- and eightmonth-old infants and showed that seroconversion was significantly lower for the 3 serotypes both after the first and the second dose of IPV-f. Comparing IPV-f versus IPV-C, they report that with the first dose, seroconversion for serotype 1 was $16.6 \%$ vs $46.4 \%$ respectively $(\mathrm{p}<0.001)$, while for serotype 2 it was $47.1 \% \mathrm{vs}$ $62.7 \%(p=0.008)$; and for serotype $3,14.6 \%$ vs $32 \%$ $(\mathrm{p}<0.001)$. With the second dose, IPV-f induction of serotype 1 seroconversion was $92.4 \%$ versus $100 \%$ with IPV-C ( $\mathrm{p}=0.01)$, for serotype 2 , seroconversions were $96.4 \%$ vs $100 \%(\mathrm{p}=0.41)$, and for serotype $3,91.8 \% \mathrm{vs}$. $99 \%(\mathrm{p}=0.018)$.

In a WHO-sponsored study in Oman, Mohammed AJ et $\mathrm{a}^{18}$ conducted a study using a different administration schedule based on age. They randomized 373 children into two groups and they were all vaccinated at 2, 4 and 6 months. One group $(n=187$ children) received IPV-f (i.e., $0.1 \mathrm{ml}$, accounting for one fifth of a full dose) intradermally through a special device (Bioejector 2000, Bioject), and the other group $(n=186)$ received IPV-C intramuscularly. This study showed that when the children were vaccinated at 2, 4 and 6 months of age, intradermal IPV-f induced seroconversion levels similar to those obtained with IPV-C, but it gave rise to significantly lower titers. After completing the three-dose program, the 30-day seroconversion rates for poliovirus serotypes 1, 2 and 3 were $97.3 \%, 95.7 \%$ and $97.9 \%$, respectively in the IPV-f group, while a $100 \%$ seroconversion was attained for the 3 serotypes in the IPV-C group ( $p=0.01$ for poliovirus type 2 ; the differences for poliovirus types 1 and 3 were not significant). Median antibody titers were also significantly lower in the fractional IPV group than in the complete IPV group $(\mathrm{p}<0.001$ for the three serotypes) (Table 2).

On the other hand, in the Philippines Cardona-Carlos $\mathrm{J}$ et al, ${ }^{19}$ administered intradermal IPV-f with syringes and 13-mm needles, $30 \mathrm{G}$ or a dose of IM IPV-C to healthy infants at 6,10 and 14 weeks, followed by a booster at 15-18 months of age. Although the levels of seroconversion after 3 doses of IPV-f as well as after the 


\begin{tabular}{|c|c|c|c|}
\hline & \multicolumn{2}{|c|}{$\%$ of seroconversion } & \multirow[t]{2}{*}{ p Value } \\
\hline & $\begin{array}{l}\text { Fractional dose } \\
\qquad n=187\end{array}$ & $\begin{array}{l}\text { Complete dose } \\
n=186\end{array}$ & \\
\hline \multicolumn{4}{|c|}{ After 3rd dose (7 months) } \\
\hline Polio 1 & $97.3 \%$ & $100 \%$ & 0.07 \\
\hline Polio 2 & $95.7 \%$ & $100 \%$ & 0.01 \\
\hline Polio 3 & $95.7 \%$ & $100 \%$ & 0.13 \\
\hline \multicolumn{4}{|c|}{ Antibody titers (7 months) } \\
\hline Polio 1 & $228(144-287)$ & $724(575-912)$ & $<0.001$ \\
\hline Polio 2 & $287(228-456)$ & $1149(912-1149)$ & $<0.001$ \\
\hline Polio 3 & $362(287-456)$ & $\geq 1448(\geq 1448-\geq 1448$ & $<0.001$ \\
\hline
\end{tabular}

Adapted from Mohammed A et al. Fractional Doses of Inactivated Poliovirus Vaccine in Oman ${ }^{18}$. two doses of bOPV (80.3\%), compared to the scheme that used two doses of IPV-C followed by one dose of bOPV $(98.3 \%)$, or the scheme in which all doses were IPV-C $(100 \%)(p<0.0001)$. The significance of having an annual cohort of $20 \%$ of vaccinated children (the total percentage would be probably higher given the suboptimal vaccination coverage in Latin America) with no poliovirus 2 seroconversion is worrisome and should be adequately analyzed.

\section{Adverse effects}

Several studies have documented a higher rate of adverse effects - mainly erythema and induration - using the intradermal route. Resik S. et al ${ }^{17}$, identified the presence of erythema and induration at the administration site (intradermal) of the IPV-f vaccine in $30 \%$ and $7 \%$, respectively. Adverse effects after the first dose in this group were significantly higher than those observed in subjects vaccinated with (intramuscular) IPV-C; in this latter group, the presence of erythema and induration at the administration site was observed in $2 \%(<0.001)$ and $1.3 \%(p=0.03)$, respectively. The same frequency of adverse effects continued to be observed with the second dose of IPV-f. In the Philippines, Cardona-Carlos J et al (19), also identified higher rates of erythema in patients vaccinated with IPV-f, both after the first and the second dose when compared to IPV-C, i.e., $69.5 \%$ vs $29 \%$ for the first dose and $38.4 \%$ vs $11.5 \%$ for the second.

\section{Programmatic challenges}

In the virtual ad-hoc Meeting of the 2017 TAG, $\mathrm{PAHO} / \mathrm{WHO}$ declare that "the intradermal administration of vaccines is more difficult than the intramuscular route; it is important for health workers to receive timely training and oversight to ensure safe vaccination. For the introduction of an IPV-f scheme, the planning, training and supervision programs should consider the updating of the documentation systems".

The same document states "According to the WHO open vial policy, multi-dose vials of IPV can be used for up to 28 days. IPV-f should be administered with a $0.1 \mathrm{ml} 27 \mathrm{G} \mathrm{3/8}$ syringe, the same syringe used by some countries to administer $B C G$. Some countries administer $B C G$ in $0.05 \mathrm{ml}$ syringes, so they do not have $0.1 \mathrm{ml}$ syringes. According to Revolving Fund data, the $0.1 \mathrm{ml}$ syringe typically used to administer BCG is very likely not available in most countries".

One of the additional challenges in the potential adoption of the intradermal IPV-f vaccination strategy is that it is more complex than delivering IM vaccines ${ }^{23,24}$. The staff needs to be specifically trained, since there is no experience with intradermal vaccines (e.g., BCG) in most community-based vaccination centers, given that 
in most LAC countries, BCG is applied in maternity hospitals and it is time-consuming. Health care workers should be well trained in the technique, as incorrect administration can lead to adverse events or a lower rate of seroconversion. In general, when using the intradermal route, it is difficult to determine the volume of vaccine administered accurately, due to leakage at the injection site $^{21}$. The use of special devices for intradermal administration may contribute to facilitate the implementation of this strategy. However, the use of these devices can increase costs compared to the intramuscular administration of the vaccine ${ }^{25}$.

Regarding the availability of needle-free devices (e.g., disposable syringe jet injectors) and needle devices (i.e., hollow microneedles and adapters that attach to the center of the intradermal needle and syringe), studies in Cuba have recently shown that seroconversion depends on the device employed; thus, when comparing the immune response using two needle-free disposable syringe injectors (Tropis and Biojector 2000) versus the BCG needle and syringe, it was found to be similar against the 3 poliovirus serotypes. However, with a third injector (Bioject) the immune response was significantly lower; with seroconversion rates for poliovirus type 2 of $22.6 \%$ compared to $49 \% \%^{24}$.

There are also other concerns regarding the introduction of the IPV-f. Delivering an intradermal vaccine requires better trained personnel, and it is more time consuming than the intramuscular injection. In addition, it implies adding one more shot into an already busy infant vaccination schedule. Previous experience in the Americas also reveals that additional doses of specific vaccines (especially injectable), entail the risk of reducing vaccination coverage rates with the second dose; as a result, these children are left with one single dose of the vaccine. Furthermore, the supply and availability of this two-dose intradermal application vaccine cannot be guaranteed.

\section{Discussion}

After reviewing the final report of PAHO's GTA and the information available, the working group had an extensive debate regarding the shortage of IPV that hits some areas of the world, (but spares the US and Europe) and decided to address the five issues below:

- Immunogenicity of the IPV-C vs IPV- f.

- Reactogenicity - adverse events.

- Program challenges.

- Alternative options.

- Ethical aspects.

The working group concluded the following:

\section{Regarding the Immunogenicity of the IPV-C vs. IPV-f:}

- Regarding IPV-C, studies conducted in Latin America have shown that vaccination schemes that include a single dose of IPV followed by two doses of bOPV do not elicit an adequate immunogenic response when compared to a 2-dose schedule of IPV-C followed by one dose of bOPV or a 3-dose IPV-C scheme.

- The existing studies show that the fractional form of IPV (1/5 of the full dose) and administered intradermally yields a lower immunogenicity than that obtained with the first dose of IPV-C and similar immunogenicity with the second dose.

- Although the significance of the correlation of the protective levels of antibodies obtained with the IPV and the durability of the protection have not been defined, the geometric mean titers are higher with IPV-C compared to the titers obtained with IPV-f. This aspect is considered relevant, since in the absence of natural circulation of the virus there will be no antigenic stimuli (booster) in the environment.

- Sub-optimal vaccination coverage rate, which worsen with the second dose in most countries, could result in accumulated cohorts of susceptible subjects that would consequently be at risk of developing polio in case of subsequent exposure to a wild virus or a VDPV.

- The research studies carried out on IPV-f have been short and involve small groups of children. Their internal validity is not challenged; however, their external validity cannot be proven, since they were performed under controlled conditions, and with different intradermal delivery devices that are not comparable to each other. Furthermore, there is no comparison between the trained staff that works in research study protocols and the trained staff that works in the community. This is an important point because there are no studies supporting the conclusion that technically heterogeneous administration produces the same immune response or modifies the rate of adverse events.

\section{Regarding reactogenicity - adverse events}

- Studies have shown that the administration of IPV-f has been accompanied by a higher proportion of local adverse events, mainly induration and local erythema in $30 \%-60 \%$ vs $<5 \%$, compared to the intramuscular administration of IPV. This may have an impact on vaccination coverage rates and engender negative opinions about vaccines from anti-vaccine groups, affecting the entire vaccination campaign in the countries.

- Countries are urged to conduct comprehensive shortterm monitoring of Events Supposedly Attributable to Vaccination or Immunization (ESAVIs). 


\section{Regarding the implementation challenges}

- Intradermal application entails logistical, programmatic and operational considerations that will require adequate training and continuous supervision in order to ensure safe vaccination. It should be anticipated that the programmatic difficulties posed by intradermal application may affect current vaccination coverage rates.

- Although the immunogenic capacity and the bioactivity of the multi-dose vial of the IPV vaccine are preserved up to 28 days once the vial is opened and/ if kept under controlled conditions ${ }^{15}$, the use of the biologic in the field may lead to handling-related contamination, with the potential of ensuing infections. This risk should not be minimized. For instance, even in the case of medications that are known to preserve their bioactivity for weeks, in many hospitals the standard practice is to change bottles within 7 days of opening.

- The strategy of actively searching for unvaccinated children, usually in remote communities, may be in the hands of poorly trained individuals due to the difficulties to train staff that work in distant or rural health care units for the most part, as is sometimes the case in some areas of Latin America.

- At this point we must consider that health legislation varies between different countries, and there may be several legal barriers. In some countries, the law requires that the intradermal delivery technique be performed only by midwives or university-trained nurses. Introducing this technique may require law amendments before any such program can be implemented; this will require time and internal debate in the countries in question.

- The implementation of the IPV-f vaccine entails investing in the training of the personnel and the development and printing of manuals, things that are not always done optimally in the field.

- An important point to note about the present situation is that no cost-effectiveness studies have been carried out in the face of a temporary shortage. Therefore, the preparedness plans for the introduction of the IPV-f entails, among other things, expenses related to introducing a new vaccination schedule, which includes training, preparation of materials and the purchase of inputs for the administration of the vaccine.

\section{Regarding alternative options}

- The group recommends following the recommendations of SLIPE and ALAPE, either with the optimal schedule using four doses of IPV-C (three doses in the primary schedule plus a booster) or schedules with IPV-C in formulations combined with other immu- nogenic agents as indicated in the country's immunization program ${ }^{11}$. There are no problems or adverse events, if a child receiving combination vaccines gets more doses of IPV-C than those recommended in the primary schedule; or an alternative schedule, for the time being, while using bOPV, it is acceptable to combine the 2 or 3 first doses of IPV-C followed by one or two doses of bOPV. At the same time, the supply of vaccine combinations containing IPV must be ensured.

- Countries that have only 1 dose of IPV-C are encouraged to update their vaccination schedules as soon as possible, to include at least 2 doses of IPV-C, considering the sub-optimal immunogenic response observed with single dose schedules.

- PAHO, the ministries of health and the pharmaceutical industry are urged to maintain a dialogue and negotiate in order to increase the production of the IPV-C and thus avoid shortages.

- Finally, we do not recommend the use of IPV-f, based on the scientific evidence available regarding immunogenicity, frequency of adverse effects, and implementation difficulties; countries are also urged to analyze the existing options that include IPV-C, be it in single-dose vials, multi-dose vials or in combined vaccines that can be incorporated into vaccination schedules.

\section{Regarding the ethical aspects}

- The group of experts cannot endorse or recommend the use of a product that - as occurs with IPV-f comes with no information from the manufacturer and has not been approved by the countries' regulatory authorities. Therefore, the IPV-C vaccines currently used in the vaccination programs of Latin America should not be fractional.

- The studies show a very short-term immunogenic response, but there are no studies that show that $1 / 5$ of the applied dose of IPV-C provide the same long-term protection. There is a risk of leaving a cohort of children with poor long-term protection in the future. In addition to being susceptible, these children will be an extra burden and matter of concern for the affected countries since they will have to be re-vaccinated.

- From a human rights perspective, and particularly children's rights, the intradermal route would be violating the principle of protecting children, since it exposes them to a route of administration that causes more adverse events in terms of pain, induration and erythema. Also, the legal issue of protecting the health-care personnel is very important, since the vaccine in itself produces more side effects, and people can mistakenly attribute those effects to the technique and reduce the self-confidence of workers. 
In addition, due to the adverse events caused by intradermal application vs the IM route, the application of subsequent doses is jeopardized, compromising vaccination coverage.

- There is an ethical discussion regarding equity, as $\mathrm{PAHO} / \mathrm{WHO}$ are inducing Latin America to introduce the use of IPV-f, an option that is not being requested of countries in Europe and the United States of America, leading to a situation of precarious immunization schedules, with obvious benefits to central countries and generating a condition of inequity in world public health.

- Finally, the group of experts does not currently recommend the use of IPV-f, considering the above-mentioned factors in terms of immunogenicity, adverse events and implementation challenges, even more so when there are IPV options available at present.

\section{Final considerations}

The group of experts calls on the countries of Latin America to make joint decisions.

While there is evidence of immunogenicity with two doses of IPV-f, this evidence alone is not sufficient for decision-making. When discussing vaccinations, there are several aspects that must be considered, including those below:

- Magnitude of the problem.

- Benefits vs adverse events.

- Long-term protection.

- Cost-effectiveness.

- Feasibility of Implementation.
- Product availability.

- Social acceptance.

- Product safety.

- Equity.

- Legal and regulatory aspects of the health authority.

- Ethical aspects.

Therefore, these dimensions need to be analyzed, to enable the adoption of the best decision possible in the event of a temporary shortage.

\section{Members of the Paraguayan Society of Pediatrics Study Group \\ Rolón-Arámbulo, Roger, Luque Regional Hospital;} Campuzano de Rolón, Ana, Clinical Hospital, Asunción National University; Lovera, Dolores, Tropical Medicine Institute; Castro, Hector, Institute of Social Welfare and Acosta-Ñú Children's Hospital; Esquivel, Ida, Paraguayan Society of Pediatrics; Nissen, Julio, Paraguayan Society of Pediatrics; Araya, Araya, Tropical Medicine Institute; Garcete Lidia, Clinical Hospital, Asunción National University; Sanchez, Maria José, Military Hospital; Chirico, Mirian, Clinical Hospital, Asunción National University; Rodriguez, Mónica, Institute of Social Welfare; Bogado, Norma, Clinical Hospital, Asunción National University; Mir, Ramón, Clinical Hospital, Asunción National University; Olmedo, Raúl, Paraguayan Society of Pediatrics; Flecha, Claudia, Institute of Social Welfare; Arrechea, Ana, San Pablo Mother and Child Hospital.

\section{References}

1.- Pan American Health Organization. 2nd ad-hoc Meeting of the Technical Advisory Group on Vaccine-preventable Diseases. 10 March 2017 Washington, DC, USA.

2.- World Health Organization. The Global Polio Eradication Initiative. Polio eradication and endgame strategic plan 2013-2018. Geneva, Switzerland; 2013.

3.- Organizacion Mundial de la Salud. $41^{\circ}$ Asamblea Mundial de la Salud. Ginebra, 2 -13 de mayo de 1988. WHA41/1988/REC/1.

4.- World Health Organization. Global Polio Eradication Initiative. Available at: http:// polioeradication.org/polio-today/polio-now/ this-week/. (Acceso el 29-05-2018).

5.- Pan American Health Organization. Expanded Program on Immunization in the Americas. EPI newsletter, Bull Pan Am Health Organ. 14 ${ }^{\mathrm{a}}$ ed. Washington (DC); 1992.

6.- Pan American Health Organization. Expanded Program on Immunization in the Americas. EPI newsletter, Bull Pan Am Health Organ. 16 ed. Washington D.C.; 1994.

7.- Landaverde J M, Trumbo S P, DanovaroHolliday M C, Cochi S E, Gandhi R, et al. Vaccine-associated paralytic poliomyelitis in the post elimination era in Latin America and the Caribbean, 1992-2011. J Infect Dis 2014; 209: 1393-402. doi: 10.1093/infdis/jit602

8.- Kew O, Morris-Glasgow V, Landaverde M, Burns C, Shaw J, Garib Z, et al. Outbreak of poliomyelitis in Hispaniola associated with circulating type 1 vaccine-derived poliovirus. Science 2002; 296: 356-9. DOI:10.1126/ science. 1068284.

9.- Centers for Disease Control and Prevention. MMWR Morb Mortal Wkly Rep. Update on Vaccine-Derived Polioviruses-Worldwide, April 2011-June 2012. 2012; Sept 21, 61 (37); 741-6). https://www.cdc.gov/mmwr/preview/ mmwrhtml/mm6137a3.htm

10.- CDC. Poliovirus prevention in the United States: updated recommendations of the Advisory Committee on Immunization
Practices (ACIP). MMWR 2000;49(No. RR-5): 1-22. https://www.cdc.gov/mmwr/preview/ mmwrhtml/rr4905a1.htm

11.- Falleiros-Arlant LH, Avila-Agüero ML, Brea del Castillo J, Mariño C. El desafío del cambio de la vacuna inactivada contra poliomielitis en América Latina. Declaración de la Sociedad Latinoamericana de Infectología Pediátrica (SLIPE). Rev Chilena Infectol 2014; 31: 590-96. http://dx.doi.org/10.4067/S071610182014000500012.

12.- Organización Mundial de la Salud. Poliomielitis. http://www.who.int/mediacentre/ factsheets/fs114/en/. (Accessed on 02-06-18).

13.- Wallace GS, Seward JF, Pallansch MA. Interim CDC guidance for polio vaccination for travel to and from countries affected by wild poliovirus. MMWR Morb Mortal Wkly Rep 2014; 63: 591-4). PMCID: PMC4584713.

14.- PAHO. Technical Advisory Group on Vaccinepreventable Diseases (TAG). XXIV Meeting 12-14 July 2017.

15.- PAHO. Background and Technical Rationale 
for Introduction of one dose of Inactivated Polio Vaccine (IPV) in Routine Immunization Schedule. January 2015.

16.- World Health Organization. Poliomyelitis: intensification of the global eradication initiative. Geneva, Switzerland: World Health Organization; 2012

17.- Resik S, Tejeda A, Sutter R W, Díaz M, Sarmiento L, Alemañi N, et al. Priming after a fractional dose of inactivated poliovirus vaccine. N Engl J Med 2013; 368:416-24. DOI: 10.1056/NEJMoa1202541.

18.- Mohammed A, Al Awaidy S, Bawikar S, Kurup P J, Elamir E, Shaban M M, et al. Fractional doses of inactivated poliovirus vaccine in Oman. N Engl J Med 2010; 362: 2351-9. DOI: 10.1056/NEJMoa0909383.

19.- Cadorna-Carlos J, Vidor E, Bonnet M C. Randomized controlled study of fractional doses of inactivated poliovirus vaccine administered intradermally with a needle in the Philippines. Intern J Infect Dis 16 (2012) e110-e116. doi: 10.1016/j.ijid.2011.10.002

20.- Resik S, Tejeda A, Díaz M, Okayasu H, Sein C, Molodecky N A, et al. Boosting immune responses following fractional-dose inactivated poliovirus vaccine: a randomized, controlled trial. J Infect Dis 2017: 215.175-82.

DOI: 10.1093/infdis/jiw492.

21.- Bibby J, Saidu Y, Umesi A, MonekeAnyanwoke N, Bashorun A O, Badjie Hydara $\mathrm{M}$, et al. The immunogenicity of fractional intradermal doses of the inactivated poliovirus vaccine is associated with the size of the intradermal fluid bleb. Clin Infect Dis 2017; 65:851-54. DOI: $10.1093 / \mathrm{cid} / \mathrm{cix} 381$

22.- O'Ryan M, Bandyopadhyay A, Villena R, Espinoza M, Novoa J, Weldon W C, et al. Inactivated poliovirus vaccine given alone or in a sequential schedule with bivalent oral poliovirus vaccine in Chilean infants: a randomised, controlled, open-label, phase 4, non-inferiority study. Lancet Infect Dis 2015; 15:1273-82. DOI: https://doi.org/10.1016/ S1473-3099(15)00219-4.

23.- Okayasu H, Sein C, Chang Blanc D, González A R, Zehrung D, Jarrahian C, et al. Intradermal administration of fractional doses of inactivated poliovirus vaccine: a dose-sparing option for polio immunization. J Infect Dis 2017; 216 (S1): S161-7. DOI: 10.1093/infdis/jix038

24.- Hickling J K, Jones K R, Friede M, Zehrung D, Chenc D, Kristensenc D. Intradermal delivery of vaccines: potential benefits and current challenges. Bull World Health Organ 2011; 89:221-226. DOI: 10.2471/BLT.10.079426.

25.- Griffiths U K, Santos A C, Nundy N, Jacoby E, Matthias D. Incremental costs of introducing jet injection technology for delivery of routine childhood vaccinations: comparative analysis from Brazil, India, and South Africa. Vaccine 2011; 29:969-75. DOI: 10.1016/j. vaccine.2010.11.038 